\title{
APLICAÇÁO DO PLANEJAMENTO EXPERIMENTAL NO MÉTODO DE ELETRO-ÇOAGULAÇÃO PARA A REMOÇÃO DE MATÉRIA ORGÂNICA DE EFLUENTES FRIGORÍFICOS
}

\author{
Aline Roberta de Pauli ${ }^{I}$ \\ Fernando Rodolfo Espinoza-Quinones ${ }^{1}$ \\ Aparecido Nivaldo Módenes ${ }^{l}$ \\ Carlos Eduardo Borba ${ }^{1}$ \\ Paulo Sergio Theodoro ${ }^{I}$
}

\begin{abstract}
Resumo: Este trabalho tem por objetivo otimizar o processo da eletro-coagulação (EC) no tratamento de efluentes industriais com alta carga orgânica. Para tanto, foi construído um reator de eletro-coagulação com 6 placas de alumínio paralelas e equidistantes e volume efetivo de $800 \mathrm{~mL}$, tendo como variáveis de operação o pH inicial, o tempo de eletrólise e a intensidade de corrente elétrica. Utilizando a metodologia das superfícies de resposta, um planejamento fatorial $3^{3}$ dos experimentos de EC foi conduzido para a obtenção dos parâmetros ótimos do reator. As variáveis de resposta para avaliar a eficiência do processo foram porcentagem de remoção de cor, DQO e turbidez. A condição ótima do processo foi encontrada para valores de $\mathrm{pH}$, tempo de eletrólise e corrente de 5, 52,5 min e $4 \mathrm{~A}$, respectivamente, obtendo reduçóes de DQO, cor e turbidez em torno de 87, 94 e 98\%, respectivamente. O tratamento de eletro-coagulação de efluente industrial de frigorífico apresentou um melhor custo benefício do que o método convencional, indicando que o método EC é uma alternativa promissora em aplicaçôes industriais.
\end{abstract}

Palavras-chaves: Planejamento fatorial, Efluentes frigoríficos, Eletro-coagulação.

Abstract: In this work an optimized electro-coagulation process was applied on the food-processing industrial effluent treatment in order to reduce the high organic load. A lab-scale electro-coagulation reactor consisting of a set of six equidistant aluminum plates within a $1 \mathrm{~L}$ container was built and its performance depending on three important variables: effluent initial $\mathrm{pH}$, electrolyze time and electric current was also evaluated on the basis of the Response Surface Methodology. A $3^{3}$ factorial design on the EC experiments was performed, optimizing the reactor operating variables according to the best response of chemical oxygen demand (COD), color and turbidity removals. Second-order models representing the response variables were validated by ANOVA. The reactor optimal performance was achieved at 52.5 min electrolysis time, 4 A electric current and 5 initial $\mathrm{pH}$. Under these conditions $87 \%$ COD, 94\% color, and $98 \%$ turbidity were removed. The EC treatment of food-processing industrial effluent proved to provide a cost-effective than the conventional one, indicating clearly that the method of electro-coagulation is very promising alternative for industrial applications.

Key-words: Factorial design, Fridge effluent, Electro-coagulation.

\footnotetext{
1 Programa de Pós-graduação Stricto Sensu em Engenharia Química da Universidade Estadual do Oeste do Paraná - UNIOESTE. Rua da Faculdade, 645. Jardim La Salle. CEP 85902-000. E-mail:borba_deq@yahoo.com.br.
} 


\section{INTRODUÇÃO}

$\mathrm{Na}$ indústria frigorífica, devido às diversas etapas do processamento, o consumo de água potável é elevado. A água residuária de uma indústria de frangos, originada da limpeza (frangos, equipamento e piso), escaldagem e resfriamento, possui elementos como sangue, penas, pele e gordura, que a tornam imprópria para reuso devido à alta carga poluente. Esse efluente se caracteriza por possuir elevada Demanda Química de Oxigênio (DQO), cor e turbidez, exigindo da indústria de processamento de alimentos a aplicaçáo de tratamentos para o posterior descarte. Na maioria dos casos, esse efluente é submetido a um tratamento biológico em lagoas de estabilizaçáo ou em processos de lodos ativados, para então, o efluente ser lançado no meio ambiente (Silva, 2005).

Segundo Crespilho e Rezende (2004), vários pesquisadores estáo desenvolvendo tratamentos com reatores eletroquímicos para a descontaminação de diversos tipos de efluentes industriais. Neste processo, para desestabilizar as impurezas presentes nos efluentes faz-se necessário alterar suas características físico-químicas, por meio da passagem de corrente elétrica, liberando íons do material do eletrodo utilizado (geralmente alumínio ou ferro) que propiciam reações químicas com a formação de espécies hidrolisadas. As etapas de hidrólise do alumínio são apresentadas nas Equaçôes 1 a 4 .

Oxidação do $\mathrm{Al}$ sólido (reação anódica):

$\mathrm{Al} \rightarrow \mathrm{Al}_{(\mathrm{aq})}^{+3}+3 \mathrm{e}^{-}$

Solvatação do cátion:

$\mathrm{Al}_{(\text {aq })}^{+3}+6 \mathrm{H}_{2} \mathrm{O} \rightarrow \mathrm{Al}\left(\mathrm{H}_{2} \mathrm{O}\right)_{6}^{+3}$

Formação do agente coagulante:

$$
\mathrm{Al}\left(\mathrm{H}_{2} \mathrm{O}\right)_{6}^{+3} \rightarrow \mathrm{Al}(\mathrm{OH})_{3(s)}+3 \mathrm{H}^{+}+3 \mathrm{H}_{2} \mathrm{O}
$$

Reaçôes secundárias:

$$
\mathrm{nAl}(\mathrm{OH})_{3} \rightarrow \mathrm{Al}_{\mathrm{n}}(\mathrm{OH})_{3 \mathrm{n}(\mathrm{s})}
$$

$\mathrm{Na}$ última década, a técnica de eletro-coagulação (EC) recebeu um interesse renovado para aplicaçóes industriais. Dada sua simplicidade inerente de projeto e operação, juntamente com a crescente necessidade de sistemas eficientes no tratamento de águas residuárias, a electro-coagulação tem sido aplicada no tratamento de efluentes in- dustriais (Palácio et al., 2009; Espinoza-Quiñones et al., 2009a; Espinoza-Quińones et al., 2009b; Borba et al., 2010, Espinoza-Quiñones et al. 2012 e Módenes et al. 2012).

Neste trabalho a técnica de electro-coagulação foi aplicada no tratamento de efluentes industriais com alta carga orgânica. A metodologia de análise estatística de superfície de resposta foi aplicada na obtenção da condição operacional ótima do reator EC. Os valores de $\mathrm{pH}$ inicial (5-8), tempo de eletrolise (30-60 min) e corrente elétrica (2-4 A) foram utilizados nas várias condições experimentais EC segundo um planejamento fatorial $3^{3}$ completo. Modelos de segunda ordem nas variáveis do reator foram propostas para descrever o conjunto de dados planejados das variáveis de resposta como DQO, cor e turbidez. Foi feito o cálculo do custo operacional nas condições ótimas do reator e comparado com os valores do tratamento convencional da própria indústria.

\section{MATERIAIS E MÉTODOS}

Foram coletados 75 litros de efluente sem nenhum tratamento prévio em uma indústria frigorífica em Toledo- Paraná, que foi preservado em laboratório conforme a metodologia descrita na norma da NBR 9898/87 (ABNT, 1987). Realizou-se a caracterização desse efluente através de análises de demanda química de oxigênio (DQO), cor e turbidez conforme a metodologia descrita no Standard Methods (APHA, 2005). A DQO foi determinada pelo refluxo fechado/método colorimétrico. A turbidez (Nephelometric Turbidity Unit, NTU) foi determinada com um turbidímetro Tecnal, modelo TB1000 e a cor foi medida utilizando um colorímetro Portable Dalatogging Spectrophotometer HACH DR/2010 com comprimento de onda de $320 \mathrm{~nm}$.

$\mathrm{O}$ reator em escala laboratorial utilizado, conforme Figura 1, é constituído por um recipiente de $1 \mathrm{~L}$ e um sistema de eletrodos composto de seis placas de alumínio com $15 \mathrm{~cm}$ de altura, $7 \mathrm{~cm}$ de largura e espessura de $1 \mathrm{~mm}$ paralelas e equidistantes em $1 \mathrm{~cm}$, com uma área efetiva de $420 \mathrm{~cm}^{2}$. O sistema de eletrodos foi conectado a uma fonte de corrente contínua de modelo Instrutemp DC Power Supply - FA 1030. A polaridade da fonte foi invertida a cada vinte minutos para evitar a passivação do eletrodo. $\mathrm{O}$ volume tratado de cada amostra foi de $800 \mathrm{~mL}$. Os parâmetros a serem 
otimizados do reator foram $\mathrm{pH}$, intensidade de corrente elétrica e tempo de eletrólise. $\mathrm{O}$ ajuste do $\mathrm{pH}$ inicial foi realizado utilizando ácido clorídrico $1 \mathrm{M}$ e hidróxido de sódio $1 \mathrm{M}$.

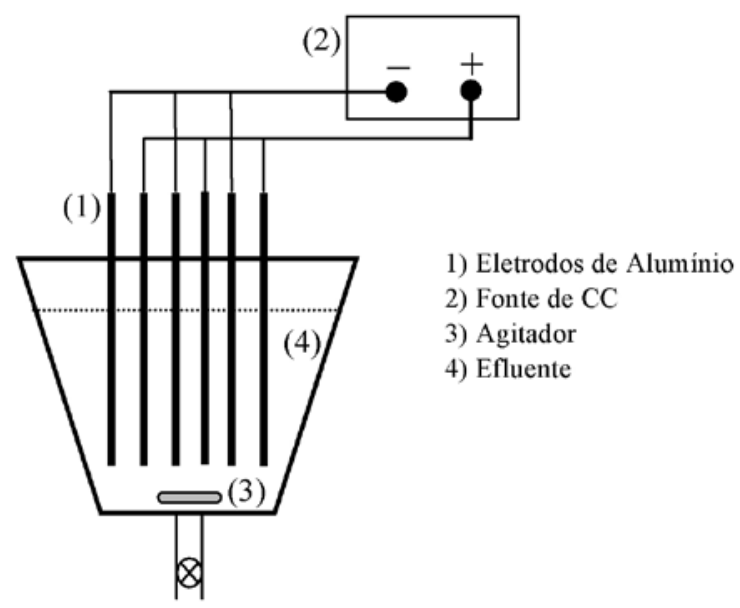

Figura 1. Esquema do reator utilizado

No planejamento fatorial $3^{3}$ os valores mínimo, intermediário e máximo para otimização dos parâmetros $\mathrm{pH}$, corrente elétrica e tempo de eletrólise no processo da eletro-coagulação encontram-se na Tabela 1, obtendo-se 27 experimentos e realizados de forma aleatória.

Tabela 1. Valores das variáveis de operação e seus níveis codificados no planejamento experimental

\begin{tabular}{|c|c|c|c|}
\hline \multirow{2}{*}{ Variáveis } & \multicolumn{3}{|c|}{ Níveis } \\
\cline { 2 - 4 } & -1 & 0 & +1 \\
\hline q1: Tempo de eletrólise (min.) & 30 & 45 & 60 \\
\hline q2: Intensidade de corrente (A) & 2 & 3 & 4 \\
\hline q3: pH inicial & 5 & 6,5 & 8 \\
\hline
\end{tabular}

Após a aplicação do processo de eletro-coagulação em cada amostra, foram realizadas análises físico-químicas de cor, turbidez e DQO para todos os 27 experimentos. Todas as análises foram realizadas em duplicata. Para representar os dados experimentais de cada variável de resposta como cor, turbidez e DQO, foi proposto um modelo (R) de segunda ordem, de acordo com a Equaçáo 5. Para validação deste modelo proposto, foi realizada a análise de variância (ANOVA).

$$
\begin{aligned}
R= & a_{0}+\sum_{i=1}^{n} a_{i} q_{j}+\sum_{i=1}^{n} \sum_{j=1}^{n} b_{j} q_{i} q_{j}+ \\
& \sum_{i=1}^{n} \sum_{j=1}^{n} \sum_{k=1}^{n} w_{j} q_{i} q_{j} q_{k}+\sum_{i=1}^{n} \sum_{j=1}^{n} v_{j} q_{i}^{2} q_{j}^{2}
\end{aligned}
$$

sendo $R$ a função resposta de cada parâmetro analisado, $q$ os parâmetros do reator $(\mathrm{pH}$, tempo de eletrólise e intensidade de corrente) e $a, b, w$ e $v$ os coeficientes significativos do modelo.

\section{RESULTADOS E DISCUSSÃO}

Antes de realizarem-se os processos de tratamento, efetuou-se a caracterização físico-química do efluente bruto. Os resultados encontrados foram de 6,4 para o $\mathrm{pH}, 89 \mathrm{PtCO}$ para a cor, 97 NTU para a turbidez e $2130 \mathrm{mg} / \mathrm{L}$ para a DQO. Durante os testes de EC observou-se que na faixa de $\mathrm{pH}$ inicial considerado (5 a 8) o pH final é deslocado para valores da regiáo alcalina devido à geração de radicais hidroxila. Como reportado por Chen (2004), os processos de EC são fortemente dependentes do $\mathrm{pH}$, devido que os íons de alumínio liberados pelos eletrodos são difíceis de agregar sob condições ácidas, enquanto os complexos tais como hidróxido de alumínio podem ser formados em $\mathrm{pH}$ alcalino.

Após a realização das análises de cor, turbidez e DQO, em duplica, calculou-se a porcentagem média de remoção de cada parâmetro para cada amostra. Esses valores, juntamente com os valores das variáveis independentes aplicadas ao tratamento são apresentados na Tabela 2. Analisando os resultados, verifica-se que as maiores taxas de porcentagem de remoção obtidas foram: 96,6\% para a cor, $100 \%$ para a turbidez e $94,3 \%$ para a DQO.

Realizando uma análise estatística baseado nos dados do planejamento fatorial $3^{3}$ (Tabela 2), verifica-se que a maior remoçáo de poluentes foi obtida operando o reator no tempo de $52,5 \mathrm{~min}$., corrente de $4 \mathrm{~A}$ e pH 5, obtendo-se uma taxa de redução máxima de $87 \%$ para DQO, 94\% para a cor e $98 \%$ para a turbidez. Os modelos de segunda ordem obtidos para as porcentagens de remoção de DQO, turbidez e cor foram validados pela análise de variância (ANOVA) cujos resultados são apresentados na Tabela 3, implicando que os modelos propostos são estatisticamente confiáveis a $95 \%(\mathrm{p}<0,05)$, de acordo com o teste da razão $\mathrm{F}\left(\mathrm{F}_{\mathrm{cal}}>\mathrm{F}_{\mathrm{tab}}\right)$. 
Tabela 2. Resultados do planejamento experimental completo $3^{3}$ para os experimentos EC.

\begin{tabular}{|c|c|c|c|c|c|}
\hline \multirow{2}{*}{$\begin{array}{l}\text { q1: Tempo de } \\
\text { eletrólise (min) }\end{array}$} & \multirow{2}{*}{$\begin{array}{l}\text { q2: Intensidade de } \\
\text { corrente }(\mathrm{A})\end{array}$} & \multirow{2}{*}{$\mathrm{q} 3: \mathrm{pH}$} & \multicolumn{3}{|c|}{ Remoção média (\%) } \\
\hline & & & Cor & Turbidez & $\mathrm{DQO}$ \\
\hline 30 & 2 & 5 & 43,8 & 96,9 & 81,3 \\
\hline 45 & 2 & 5 & 80,9 & 98,4 & 86,2 \\
\hline 60 & 2 & 5 & 62,9 & 97,0 & 83,7 \\
\hline 30 & 4 & 5 & 74,2 & 92,9 & 78,0 \\
\hline 45 & 4 & 5 & 91,0 & 98,3 & 90,2 \\
\hline 60 & 4 & 5 & 96,6 & 99,6 & 89,4 \\
\hline 30 & 3 & 5 & 73,0 & 92,8 & 85,4 \\
\hline 45 & 3 & 5 & 85,4 & 96,3 & 81,3 \\
\hline 60 & 3 & 5 & 73,0 & 95,8 & 74,0 \\
\hline 30 & 2 & 6,5 & 74,2 & 92,7 & 73,2 \\
\hline 45 & 2 & 6,5 & 56,2 & 88,0 & 82,1 \\
\hline 60 & 2 & 6,5 & 92,1 & 97,3 & 86,2 \\
\hline 30 & 4 & 6,5 & 89,9 & 96,5 & 76,4 \\
\hline 45 & 4 & 6,5 & 68,5 & 93,8 & 90,2 \\
\hline 60 & 4 & 6,5 & 86,5 & 95,1 & 71,5 \\
\hline 30 & 3 & 6,5 & 94,4 & 100,0 & 79,7 \\
\hline 45 & 3 & 6,5 & 93,3 & 95,7 & 89,4 \\
\hline 60 & 3 & 6,5 & 85,4 & 94,5 & 92,7 \\
\hline 30 & 2 & 8 & 75,3 & 94,8 & 75,6 \\
\hline 45 & 2 & 8 & 49,4 & 96,3 & 83,7 \\
\hline 60 & 2 & 8 & 47,2 & 96,7 & 56,9 \\
\hline 30 & 4 & 8 & 61,8 & 84,1 & 74,0 \\
\hline 45 & 4 & 8 & 75,3 & 96,1 & 85,4 \\
\hline 60 & 4 & 8 & 85,4 & 93,2 & 79,7 \\
\hline 30 & 3 & 8 & 64,0 & 95,9 & 81,3 \\
\hline 45 & 3 & 8 & 67,4 & 91,3 & 87,0 \\
\hline 60 & 3 & 8 & 84,3 & 96,5 & 94,3 \\
\hline
\end{tabular}

Tabela 3. Resultados da ANOVA para os modelos da remoção de turbidez, cor e DQO.

\begin{tabular}{|c|c|c|c|c|c|c|c|}
\hline \multirow{2}{*}{ Resposta } & $\begin{array}{c}\text { Fonte de } \\
\text { Variação }\end{array}$ & $\begin{array}{c}\text { Soma } \\
\text { Quadrática }\end{array}$ & $\begin{array}{c}\text { Graus de } \\
\text { Liberdade }\end{array}$ & $\begin{array}{c}\text { Média } \\
\text { Quad. }\end{array}$ & $\begin{array}{c}\text { F } \\
\text { Calc. }\end{array}$ & $\begin{array}{c}\text { F } \\
\text { Tab. }\end{array}$ & p-Valor \\
\hline \multirow{3}{*}{ Turbidez } & Regressáo & 202 & 10 & 20,18 & 3,06 & 2,2 & 0,02 \\
\cline { 2 - 8 } & Erro & 105,44 & 16 & 6,59 & & & \\
\cline { 2 - 8 } & Total SS & 307,26 & 26 & & & & \\
\hline \multirow{3}{*}{ Cor } & Regressão & 3293 & 6 & 548,83 & 4,33 & 2,47 & 0,006 \\
\cline { 2 - 9 } & Erro & 2533,23 & 20 & 126,66 & & & \\
\cline { 2 - 9 } & Total SS & 5826,24 & 26 & & & & \\
\hline \multirow{3}{*}{ DQO } & Regressão & 690 & 4 & 172,54 & 3,98 & 2,74 & \\
\cline { 2 - 8 } & Erro & 954,56 & 22 & 43,39 & & & \\
\cline { 2 - 8 } & Total SS & 1644,72 & 26 & & & & \\
\hline
\end{tabular}




\subsection{ANÁLISE DA FUNÇÃO RESPOSTA DA} COR

O modelo quadrático para a remoção da cor, validado pela ANOVA, é dada pela Equação 6. Ao nível de $95 \%$ de confiança há seis coeficientes significativos englobando as dependências lineares e quadráticas dos parâmetros operacionais do reator EC. Verifica-se que o coeficiente linear negativo $(-3,9)$ para o $\mathrm{pH}$ inicial $\left(\mathrm{q}_{3}\right)$ sugere menores valores para o $\mathrm{pH}$ para maximizar a redução da cor. No entanto, o valor positivo $(5,3)$ quando o valor do $\mathrm{pH}$ está em ordem quadrática indica maiores valores do $\mathrm{pH}$ para aumentar a porcentagem de remoção da cor. Assim, um valor intermediário desta variável é esperado para que ocorra a remoção máxima da cor. Esta influência do $\mathrm{pH}$ pode ser melhor visualizada na superfície de resposta para a cor apresentada na Figura 2.

$\mathrm{Na}$ Figura 2a, fixando o tempo em 52,5 min, verifica-se que a região de máxima remoção da cor é bem ampla, sugerindo pouca variabilidade na reduçáo da cor quando o $\mathrm{pH}$ varia de 6 a 7 e a corrente varia de 3 a $4 \mathrm{~A}$. O mesmo ocorre quando a corrente é mantida fixa em $4 \mathrm{~A}$ (Figura $2 \mathrm{~b})$, verificando-se pouca variação na taxa de remoção da cor quando o tempo varia entre 45 e 60 min., indicando pouca diferença na eficácia do tratamento neste intervalo de tempo. Desta forma, seria então viável a utilização do menor tempo, por haver menor gasto com o processo e maior rapidez no tratamento. Mantendo-se o $\mathrm{pH}$ fixo em 5 (Fig. 2c), verifica-se que a máxima porcentagem de remoção está numa região compreendida no tempo entre 50 e 60 minutos e o valor da corrente entre 3 e $4 \mathrm{~A}$.

$$
\begin{aligned}
R_{\text {Cor }}(\%)= & 75+3,5 q_{1}+8,2 q_{2}-3,9 q_{3}+ \\
& 5,3 q_{3}^{2}-5,2 q_{1}^{2} q_{3}-4,8 q_{1}^{2} q_{3}^{2}
\end{aligned}
$$

(a)

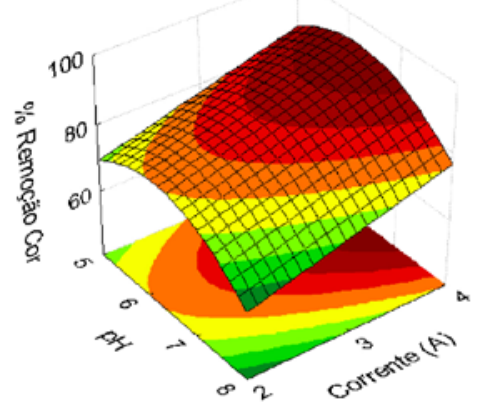

(b)

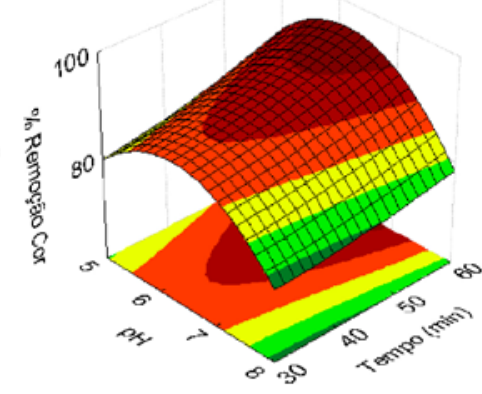

(c)

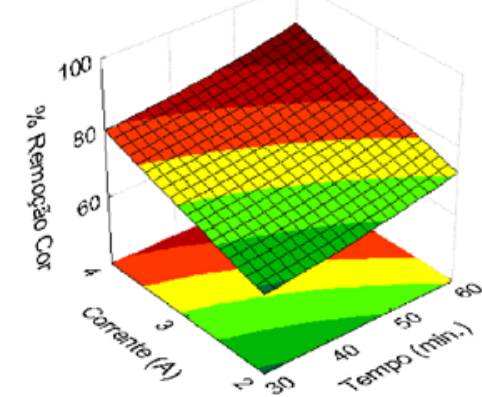

Figura 2. Superfícies de resposta para a remoção da cor, mantendo fixo (a) o tempo de eletrólise em 52,5 min; (b) a corrente em $4 \mathrm{~A}$; e (c) o pH em 5 .

\subsection{ANÁLISE DA FUNÇÃO RESPOSTA DA TURBIDEZ}

A função resposta da turbidez, validada pela ANOVA, é apresentada na Equação 7. Ao nível de $95 \%$ de confiança há dez coeficientes significativos englobando as dependências lineares e quadráticas dos parâmetros operacionais do reator EC. Verifica-se pelos coeficientes lineares negativos para a corrente $\left(\mathrm{q}_{2}\right)$ e $\mathrm{pH}$ inicial $\left(\mathrm{q}_{3}\right)$ que uma redução nos valores dessas variáveis propicia uma melhor remoção da turbidez. Uma tendência oposta pode ser inferida nos termos quadráticos da corrente elétrica e $\mathrm{pH}$ inicial, sugerindo valores intermediários. Além disso, a remoção de turbidez é incrementada quando se assumem valores maiores de tempo de eletrolise $\left(\mathrm{q}_{1}\right)$. As açóes combinadas dos parâmetros de operaçáo do reator podem ser visualizadas na Figura 3. Assim, mantendo fixo 
o pH inicial em 5 (Fig. 3a), observa-se que a máxima porcentagem de remoção está numa região compreendida no valor da corrente entre 3 e $4 \mathrm{~A}$ e no tempo entre 45 e 60 minutos, porém há pouca diferença na remoção de turbidez neste intervalo de tempo, tornando-se mais viável a utilização do tempo menor pela economia gerada. Além disso, mantendo fixa a corrente em 4 A (Fig. 3b), observa-se que a máxima porcentagem de remoção esta numa regiáo compreendida no tempo entre 40 a 60 minutos e o valor do $\mathrm{pH}$ entre 5,0 e 6,0. Novamente, nota-se que a porcentagem de remoção da turbidez é aproximadamente a mesma para o tempo entre 45 e 60 minutos. Quando o tempo de eletrolise é fixado em 52,5 min. (Fig. 3c), verifica-se que a máxima porcentagem de remoção da turbidez está numa regiáo compreendida no valor de corrente entre 3 a $4 \mathrm{~A}$ e $\mathrm{pH}$ entre 5,0 e 7,0, sendo que para esses valores de $\mathrm{pH}$ a porcentagem de remoção da turbidez é quase a mesma.

$$
\begin{gathered}
R_{\text {turdidez }}(\%)=95+1 q_{1}-0,5 q_{2}-1,3 q_{3}+ \\
1 q_{2}^{2} q_{3}-1,1 q_{1} q_{2}^{2}+1 q_{1}^{2} q_{2}-1,2 q_{1} q_{3}^{2}+ \\
1,3 q_{2} q_{3}^{2}+1 q_{2}^{2} q_{3}^{2}-1,3 q_{1}^{2} q_{3}^{2}
\end{gathered}
$$

\subsection{ANÁLISE DA FUNÇÃO RESPOSTA DA DQO}

No nível de confiança exigido ao modelo quadrático de resposta da DQO (Equação 8) e validado pela ANOVA, verifica-se a existência de poucos coeficientes significativos $(\mathrm{p}<0,05)$. A remoção de DQO é fortemente influenciada pelo aumento do tempo de eletrolise e não sofrendo influencia linear nos outros parâmetros (corrente e $\mathrm{pH}$ inicial). Analisando-se os termos quadráticos a remoção da DQO aumenta com o aumento do tempo de eletrolise e corrente elétrica. As açôes combinadas dos parâmetros de operação do reator podem ser visualizadas na Figura 4. Assim, mantendo fixo a corrente em 4 A (Fig. 4a), observa-se que a máxima porcentagem de remoção esta numa regiáo para valores de $\mathrm{pH}$ entre 5,0 e 7,0 e tempo em meio a 45 e 60 minutos. Além disso, mantendo o pH inicial em 5 (Fig. 4b) observa-se que a máxima porcentagem de remoção esta no intervalo de tempo de eletrolise entre 40 e 55 min. e o valor da corrente entre 3 e 4 A. Fixando o tempo de eletrolise em 52,5 min. a superfície de resposta para a remoção da DQO torna-se plana
(Fig. 4 c) e a máxima percentagem de remoção da DQO encontra-se no intervalo de corrente entre 3,5 a $4 \mathrm{~A}$ e $\mathrm{pH}$ entre 5 e 5,5 .

$$
\begin{aligned}
R_{D Q O}(\%)= & 82+1,3 q_{1}+3,3 q_{1}^{2}+ \\
& 2,4 q_{2}^{2}+4,1 q_{2}^{2} q_{3}
\end{aligned}
$$

(a)

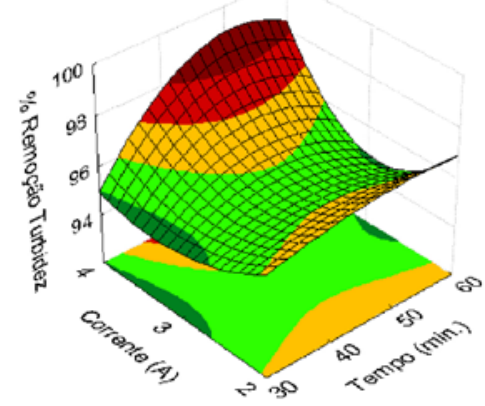

(b)

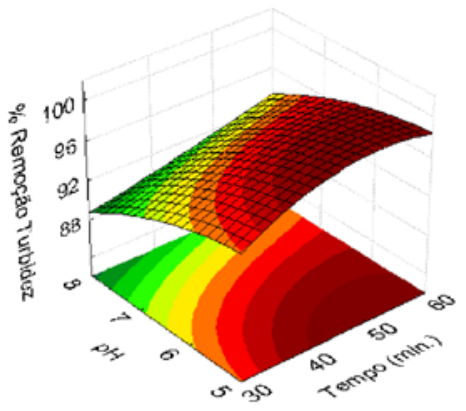

(c)

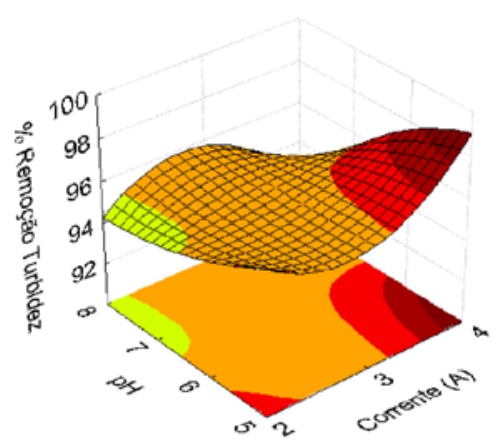

Figura 3. Superfícies de resposta para a remoção da turbidez, mantendo fixo (a) o $\mathrm{pH}$ em 5; (b) a corrente em $4 \mathrm{~A}$; e (c) o tempo de eletrólise em $52,5 \mathrm{~min}$. 
(a)

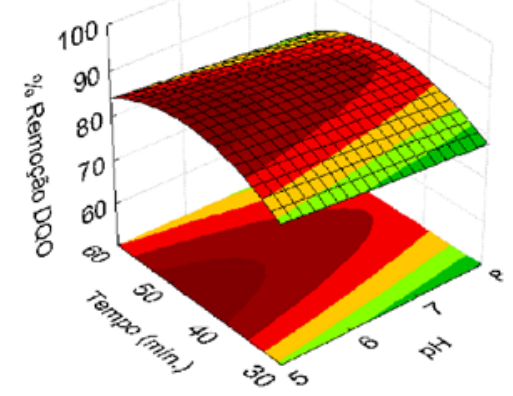

(b)

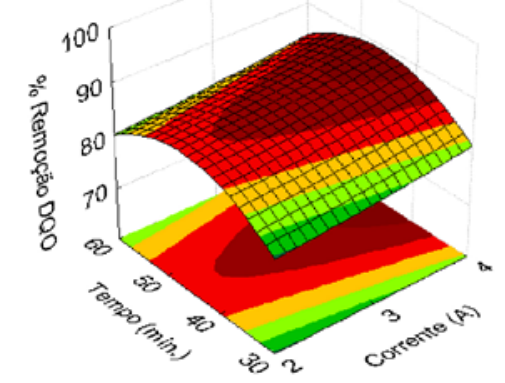

(c)

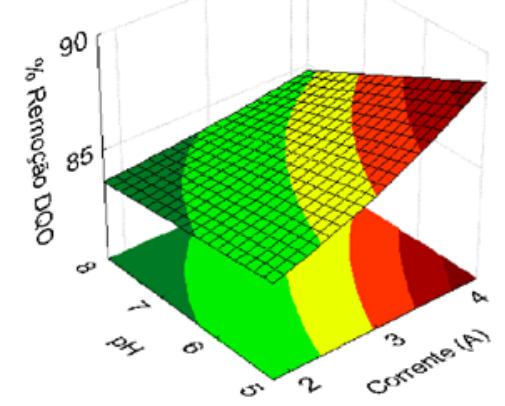

Figura 4. Superfícies de resposta para o percentual de remoção da DQO, mantendo fixo (a) o pH em 5; (b) a corrente em 4 A; e (c) o tempo de eletrólise em 52,5 min.

\subsection{ANÁLISE DE CUSTO OPERACIONAL}

O cálculo do custo elétrico (CE) foi realizado utilizando-se a Equação 9, juntamente com os valores ótimos obtidos para o $\mathrm{pH}$ inicial e a corrente elétrica do planejamento experimental, enquanto que o tempo de eletrolise foi variado entre 0 e $60 \mathrm{~min}$.

$$
C E=\frac{V i t}{V_{e f}} P E
$$

Sendo $C E$ o custo operacional da eletro-coagulação $\left(\mathrm{em} \mathrm{R} \$ / \mathrm{m}^{3}\right)$ de efluente tratado, $V$ é a tensão aplicada $(12 \mathrm{~V}), i$ a intensidade de corrente ótima (4 A), $t$ o tempo de eletrólise (em h), $V_{e f} \mathrm{o}$ volume total de efluente tratado $(0,8 \mathrm{~L})$ e $P E$ o custo da eletricidade industrial ( $\$ 0,21 / \mathrm{kWh})$.
$\mathrm{O}$ custo dos materiais $(C M)\left(\mathrm{em} \mathrm{R} \$ / \mathrm{m}^{3}\right)$, leva em conta a massa máxima possível de alumínio teoricamente dissolvida pelo ânodo por $\mathrm{m}^{3}$ de efluente tratado, como apresentado pela Equação 10. Enquanto que o custo total de operação é dado pela Equação 11.

$$
\begin{aligned}
& C M=\frac{M i t}{n F V e_{f}} P M E \\
& C T O=C E+C M
\end{aligned}
$$

Sendo PME o preço do material de eletrodo (R\$ 9,5/kg), $M$ a massa molar relativa do alumínio $(\mathrm{kg} / \mathrm{mol}), i$ a corrente elétrica $(\mathrm{em} \mathrm{A}), t$ o tempo de eletrólise (s), $n$ o número de elétrons envolvidos na reação de oxidação/redução e $F$ a constante de Faraday (96.500 C/mol).

Os custos do tratamento pela técnica da eletro-coagulação para cada diferente tempo de operaçáo empregado juntamente com o custo do tratamento convencional (fornecido pela indústria, $\left.\mathrm{R} \$ 12,0 / \mathrm{m}^{3}\right)$ são apresentados na Figura 5.

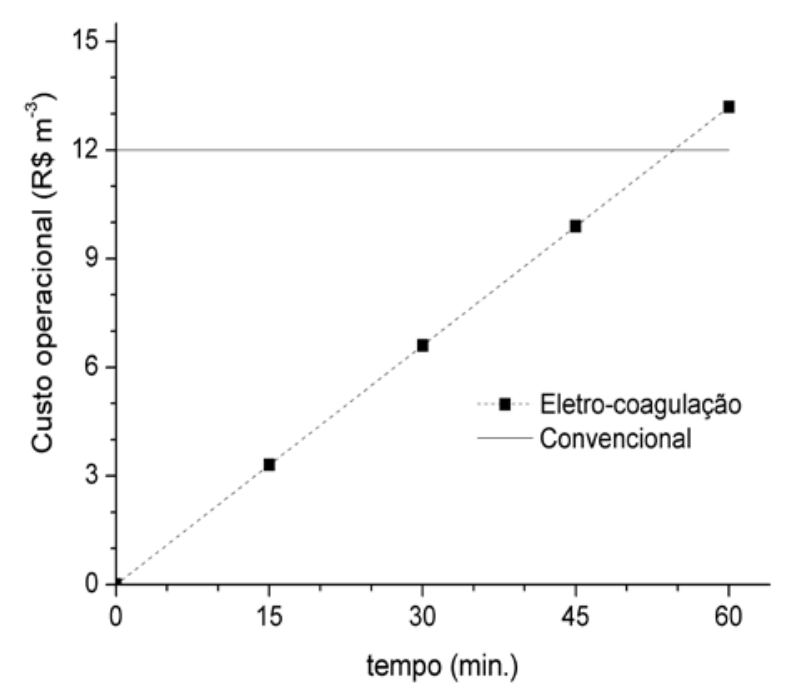

Figura 5. Comparação entre os custos de processos de tratamento de efluentes convencionais e o custo do processo de eletro-coagulação no tratamento de efluente frigorífico

Ao analisar a Figura 5, verifica-se que até o tempo de $55 \mathrm{~min}$. o processo de eletro-coagulação mostrou-se mais econômico do que o tratamento convencional. Em análise preliminar do efluente tratado, fornecido por uma indústria frigorífica em Toledo-Paraná, a remoção de DQO pelo 
método convencional foi de $96 \%$, resultado levemente melhor do que o do método de eletrocoagulaçáo para o tempo de tratamento de 55 min.

Analisando a Equaçáo 8, modelo de segunda ordem para a porcentagem de remoçáo de DQO obtido pela análise estatística dos dados do planejamento experimental, mantendo fixos a corrente em 4 A (q2) e o pH inicial em 5 (q3), verifica-se que para alcançar o mesmo resultado que o tratamento convencional na redução da DQO o processo de eletro-coagulação necessita de 70 min. de operaçáo, acarretando um custo de operação de $\mathrm{R} \$ 15,39 / \mathrm{m}^{3}$ de efluente. A grande vantagem na utilização do processo de eletro-coagulação está na reduçáo do tamanho da estação de tratamento e na eliminaçáo de produtos químicos normalmente empregados em grandes quantidades nos métodos convencionais de tratamentos.

\section{CONCLUSÃO}

Utilizando-se a metodologia de superfícies de resposta, o tratamento de efluente industrial com elevada carga orgânica foi otimizado pela aplicação de um planejamento experimental completo $3^{3}$. Os modelos de segunda ordem para a DQO, cor e turbidez foram validados pela ANOVA. A máxima eficiência na remoção da DQO, cor e turbidez foi encontrada para os valores de 5 para o $\mathrm{pH}$ inicial, $4 \mathrm{~A}$ para a corrente elétrica e 52,5 min para o tempo de eletrolise, obtendo-se uma taxa de redução máxima de $87 \%$ para DQO, $94 \%$ para a cor e $98 \%$ para a turbidez. Contudo, pode-se trabalhar com tempo de eletrólise menor que 52,5 min., porém com uma pequena diminuição na eficiência de remoção das variáveis de resposta do tratamento EC. Considerando tempos de eletrolise abaixo de $55 \mathrm{~min}$. conseguem-se custos operacionais do tratamento EC bem menores do que o custo real do tratamento convencional aplicado pela indústria de processamento de alimentos. Além do baixo custo operacional obtido pela técnica EC, as porcentagens de remoção da DQO por ambos os métodos (EC e convencional) são muito similares. No entanto, pela aplicação da técnica de eletro-coagulação evita-se o consumo elevado de reagentes empregados no tratamento convencional. Assim, o tratamento de eletro-coagulação de efluente industrial de frigorífico se mostrou apresentar um melhor custo benefício do que o método convencional, indicando que o método EC é uma alternativa promissora em aplicaçóes industriais.

\section{REFERÊNCIAS}

ABNT - Associação Brasileira de Normas Técnicas. NBR 9898/87 Preservação e técnicas de amostragem de efluentes líquidos e corpos receptores - Procedimento.

APHA, AWWA, WPCF. 2005. Standard Methods for the Examination of Water and Wastewater. 21st ed., American Public Health Association, Washington, DC.

BORBA, F.H., MANENTI, D.R., MÓDENES, A.N., MORA, N.D., ESPINOZA-QUIÑONES, F.R., PALÁCIO, S.M., YASSUE, P.H., NASCIMENTO, R. 2010. Avaliação da eficiência da técnica de eletro-floculaçáo no tratamento de efluentes de indústrias de subprodutos avícolas. Estudos Tecnológicos. v.6, pp.36-47.

CHEN, G. 2004. Electrochemical technologies in wastewater treatment. Separation and Purification Technology. v.38, pp. 11-41.

CRESPILHO, F.N., REZENDE, M.O.O. Eletroflotação: Princípios e Aplicaçôes. Editora Rima, São Carlos, SP, 2004.

ESPINOZA-QUIÑONES, F. R., FORNARI, M. M. T., MÓDENES, A. N., PALÁCIO, S. M., SILVA Jr., F. G., SZYMANSKI, N., KROUMOV, A. D., TRIGUEROS, D. E. G. 2009a. Pollutant removal from tannery effluent by electro-coagulation. Chemical Engineering Journal. v.151, pp. 59-65.

ESPINOZA-QUIÑONES, F. R., FORNARI, M. M. T., MÓDENES, A. N., PALÁCIO, S. M., TRIGUEROS, D. E. G, BORBA, F. H. SZYMANSKI, N., KROUMOV, A. D. K. 2009 b. Electrocoagulation efficiency of the tannery effluent treatment using aluminium electrodes. Water Science \& Technology. v.60, pp. 2173-2185.

ESPINOZA-QUIÑONES, F. R., MÓDENES, A. N., THEODORO, P. S., PALÁCIO, S. M., TRIGUEROS, D. E. G., BORBA, C. E., ABUGDERAH, M. M., KROUMOV, ALEXANDER 
D. 2012. Optimization of the Iron Electro-Coagulation Process of $\mathrm{Cr}, \mathrm{Ni}, \mathrm{Cu}$, and $\mathrm{Zn}$ Galvanization By-Products by Using Response Surface Methodology. Separation Science and Technology, v. 47, pp. 688-699.

MÓDENES, A. N., ESPINOZA-QUIÑONES, F.R., BORBA, F.H., MANENTI, D.R. 2012. Performance evaluation of an integrated photoFenton Electrocoagulation process applied to pollutant removal from tannery effluent in batch system. Chemical Engineering Journal, v.197, pp. $1-9$.

SILVA, H. L. B. 2005. Uso de membranas microporosas no tratamento de efluentes de um frigo- rífico de abate de aves. Dissertação de Mestrado apresentada na Faculdade de Engenharia Química da Universidade Federal de Santa Catarina.

PALÁCIO, S. M., ESPINOZA-QUIÑONES, F. R., MÓdENES, A. N. OLIVEIRA, C. C., BORBA, F. H., SILVA Jr, F. B. 2009. Toxicity assessment from electro-coagulation treated-textile dye wastewaters by bioassays. Journal of Hazardous Materials. v.172, pp. 330-337.

VALENZUELA, J. Tratamento de efluentes em indústrias galvanotécnicas. Editora Páginas e letras, São Paulo, SP, 2008.

Artigo submetido em 30/11/2011, aceito em 17/08/2012 